\title{
The electro-optical characteristics of the AZO/Mo/AZO transparent conductive film on PC substrate
}

\author{
Tien-Chai Lin ${ }^{1}$, Wen-Chang Huang ${ }^{2,}$, Fu-Chun Tsai ${ }^{2}$ \\ ${ }^{1}$ Department of Electrical Engineering, Kun Shan University, Tainan, Taiwan \\ ${ }^{2}$ Department of Electro-Optical Engineering, Kun Shan University, Tainan, Taiwan \\ Email address: \\ tienchai@mail.ksu.edu.tw (Tien-Chai Lin),wchuang@mail.ksu.edu.tw (Wen-Chang Huang)
}

\section{To cite this article:}

Tien-Chai Lin, Wen-Chang Huang, Fu-Chun Tsai. The Electro-Optical Characteristics of the AZO/Mo/AZO Transparent Conductive Film on PC Substrate. International Journal of Materials Science and Applications. Vol. 3, No. 5, 2014, pp. 205-209.

doi: $10.11648 /$ j.ijmsa.20140305.21

\begin{abstract}
The triple-layered transparent conductive oxide thin films, AZO/Mo/AZO (OMO) structure, is present in the paper. The OMO film is deposited on polycarbonate (PC) substrates by a co-sputtering process at room temperature. Metal Mo is chosen because of its chemical stable and low resistivity. The OMO film shows a great reduction of resistivity through the addition of Mo layer. The dependence of the AZO thickness of the electro-optical characteristic to the OMO film is also discussed. A best figure of merit (FOM) of $1.56 \times 10^{-5} \Omega^{-1}$ with a sheet resistance of $530 \Omega / \square$ and average transmittance of $62 \%$ is found at the OMO film of $130 \mathrm{~nm}$ thickness. At this condition, the AZO film shows a low resistivity due to its excellent crystallinity.
\end{abstract}

Keywords: Transparent Conductive Oxide, $\mathrm{ZnO}$, Mo, Sheet Resistance, Transmittance

\section{Introduction}

Transparent conductive oxide material (TCO), zinc oxide $(\mathrm{ZnO})$, is popular for its applications in many fields, such as liquid crystal display, thin film solar cells, organic light emitting diode, and plasma display panels. This is due to the material because it shows the advantages of low cost, non-toxicity, high transparency in visible range and reaction stability in hydrogen plasma [1]. $\mathrm{ZnO}$ has a wide and direct bandgap of $3.37 \mathrm{eV}$ and a large exciton binding energy of $60 \mathrm{meV}$. It shows n-type characteristic at its undoped situation. The conductivity of the $\mathrm{ZnO}$ film can be improved by adding dopant elements such as $\mathrm{Al}[2], \mathrm{Ga}[3]$, $\operatorname{In}[4], \mathrm{Mo}[5]$ and co-doped $\mathrm{Al}$ and $\mathrm{F}[6]$. However, in a practical application the resistivity of the $\mathrm{Al}$ doped $\mathrm{ZnO}$ (AZO) thin films is now low enough and limits the use of the transparent conductive electrode. To solve the problem without the sacrifice of the transparency of the film, the development of a new material or new scheme of film is needed.

It is well known that metal film shows a good conductivity but a low transparency in the visible range at a wavelength of 380-780 $\mathrm{nm}$. So the structures which combine TCO with a thin metal layer to achieve a stacked layer with low resistivity and high transparency have been developed. The stack-layered structures such as $\mathrm{Au} /$ indium-tin oxide (ITO) [7, 8] and $\mathrm{Cu} / \mathrm{AZO}$ [9] bi-layer are reported and show good electrical conductivity. However, these structures lead to another problem of low transparency due to the reflection of the metal layer. The tri-layered structure, TCO/metal/TCO, could effectively suppress the reflection from the metal layer in the visible range and achieve selective transparent effects. Therefore, the conducted researches of tri-layer TCO/metal/TCO films became of interest. The tri-layered structures such as ITO/metal/ITO[10-12], $\quad \mathrm{ZnO} / \mathrm{Cu} / \mathrm{ZnO}[13] \quad$ and $\mathrm{ZnO} / \mathrm{Ag} / \mathrm{ZnO}[14]$ were reported. They showed that the electro-optical characteristics of the OMO films heavily depended on the thickness of each layer. The optoelectronic devices include thin-film transistors (TFTs) on flexible substrates (polycarbonate (PC)) and organic devices such as organic light-emitting diodes (OLEDs) and organic photovoltaics (OPVs). In addition, many optoelectronic devices require applying $\mathrm{ZnO}$-based TCOs to replace ITO TCO film to reduce fabrication costs. Thus, the development of the OMO tri-layer structure transparent conductive layer on PC substrate is of interest and the structure, $\mathrm{AZO} / \mathrm{Mo} / \mathrm{AZO}$, is designed in the study. For the refractive index of $\mathrm{Al}$ doped $\mathrm{ZnO}(\mathrm{AZO})$ is higher than that 
of ITO. So it is chosen to be the TCO material of the tri-layered structure to effectively suppress the reflection of the metal layer. Mo is a thermally stable metal and it is a popular material in the application of CIGS solar cell. Based on the electron configurations of Mo and $\mathrm{Zn}$, the valence electron difference between $\mathrm{Mo}^{6+}$ and $\mathrm{Zn}^{2+}$ is 4 . A small amount of Mo doping can produce sufficient free carriers in order to reduce the ion scattering effects.

In this study, the tri-layered structure, $\mathrm{AZO} / \mathrm{Mo} / \mathrm{AZO}$ (OMO) was processed on a PC substrate by RF and DC magnetron sputtering. The thickness of the Mo was constant while the AZO's was varied to evaluate the effect on the OMO film. The structural, electrical and optical properties of the OMO have been studied systemically.

\section{Experimental Details}

The AZO/Mo/AZO (OMO) thin films were deposited through a co-sputtering system. Both the targets AZO ceramic $(9.999 \%$ purity, $7.2 \mathrm{~cm}$ in diameter, $0.5 \mathrm{~cm}$ thickness with $\mathrm{Al}_{2} \mathrm{O}_{3}: \mathrm{ZnO}=2: 98 \mathrm{wt} \%$ ) and $\mathrm{Mo}(99.99$ purity, $7.62 \mathrm{~cm}$ in diameter) were used to be the deposition source. A flexible polycarbonate (PC) material was chosen to be the substrate. PC substrates were first cleaned with a standard cleaning procedure: acetone, methanol and DI water in sequence for $5 \mathrm{~min}$, respectively. The distance between PC substrate and the target is about $6 \mathrm{~cm}$ and the substrate holder rotated to obtain a uniform morphology of the film during deposition. The gas ambient of the chamber is high purity argon $(99.99 \%)$. The base pressure was pumped down to $7 \times 10^{-3}$ pa and then high purity argon was inlet to the chamber, the working pressure was constant at 0.5 pa. The RF power toward AZO target was fixed at 130 $\mathrm{W}$ and the DC power toward Mo target was kept at $40 \mathrm{~W}$. The thicknesses effect of AZO on the OMO film is discussed in the study, so the samples fixed the thickness (deposition time) of Mo and changing that of AZO. The deposition time of Mo was constant at $30 \mathrm{sec}$ and that of AZO films varied as 6, 8, 10, 13 and $15 \mathrm{~min}$.

The electrical, structural and optical characteristics of the films were measured and analyzed after the deposition. $\alpha$-step was used to measure the thickness of the OMO film. The crystallinity of the film was analyzed by the X-ray diffraction (XRD) using a Ni-filtered $\mathrm{Cu} K \alpha$ source. The sheet resistances of the films were evaluated through a four-point probe. The surface morphology of the film was observed by field emission scanning electron microscopy (FE-SEM). The UV-Vis was used to evaluate the optical characteristics of the films. The Hitachi Ultraviolet-Visible 2008A Spectrophotometer was used to measure the transmittance of the films in the wavelength range between 300 to $1100 \mathrm{~nm}$.

\section{Results and Discussion}

The thickness of the OMO films with respect to the deposition time of the AZO layer is shown in Fig. 1. The values of the thickness of the OMO films are summarized in Table 1. To establish constant thickness of the Mo layer, the deposition time is fixed at $30 \mathrm{sec}$. Both the top and bottom AZO layers were obtained at the RF power of 130 $\mathrm{W}$. The deposition times varied as $6,8,10,13$ and $15 \mathrm{~min}$, respectively. The total thickness of the OMO film is $96 \mathrm{~nm}$ after 6 min-deposition and is $197 \mathrm{~nm}$ after 15 min-deposition.

Table 1. The deposition time and total thickness of each OMO layer

\begin{tabular}{lccccc}
\hline Time (min/sec/min) & $\mathbf{6 / 3 0 / 6}$ & $\mathbf{8 / 3 0 / 8}$ & $\mathbf{1 0 / 3 0 / 1 0}$ & $\mathbf{1 3 / 3 0 / 1 3}$ & $\mathbf{1 5 / 3 0 / 1 5}$ \\
\hline Thickness $(\mathrm{nm})$ & 96 & 103 & 130 & 145 & 197 \\
\hline
\end{tabular}

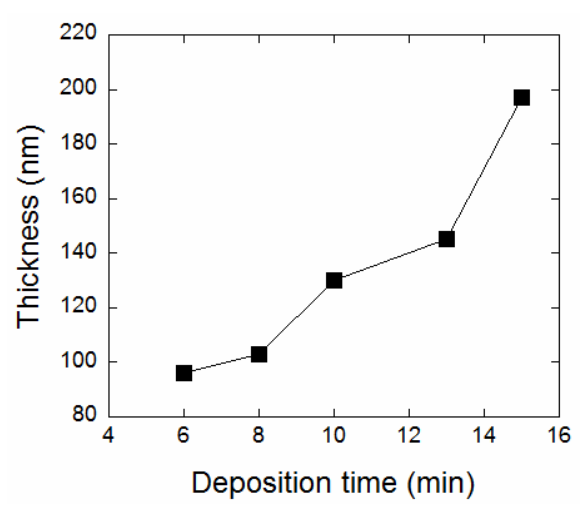

Figure 1. The thickness of the OMO film with respect to the deposited time of AZO layer.

The resistivity of the transparent conductive film is a key merit of the evaluation. Here, the sheet resistances of the OMO films were measured. The result of the evaluation is shown in Fig. 2. It is found that the sheet resistance of the OMO film decreased with the increase of the AZO thickness. The sheet resistance of the OMO film is 760 $\Omega / \square$ after $6 \mathrm{~min} / 30 \mathrm{sec} / 6 \mathrm{~min}$ deposition of each layer. It decreased to $478 \Omega / \square$ as the deposited time was increased to $15 \mathrm{~min} / 30 \mathrm{sec} / 15 \mathrm{~min}$ of each layer. According to the parallel circuit model[15], the total sheet resistance $\left(R_{s}\right)$ of the OMO layer is approximately equal to the parallel resistance of the metal $\left(R_{M o}\right)$ and AZO layers $\left(\mathrm{R}_{\mathrm{AZO}}\right)$ as followed by:

$$
\frac{1}{R_{s}}=\frac{2}{R_{A Z O}}+\frac{1}{R_{M o}} \cong \frac{1}{R_{M o}}
$$

In conclusion, the conductivity of the tri-layered film is mainly dominated by the conductivity of Mo layer. The sheet resistance of single layer AZO is $1.45 \times 10^{5}, 6.5 \times 10^{4}$, $9.5 \times 10^{3}, 5.4 \times 10^{3}$ and $3.8 \times 10^{3} \Omega / \square$ after $6,8,10,13$ and 15 min deposition, respectively. Compared with the single-layered AZO, the tri-layered OMO has a lower sheet resistance. For the OMO layer itself, the total resistance decreased as the thickness of AZO increased. This tendency is consistent with the single layered AZO. The decrease of sheet resistance of the AZO film as shown in Fig. 2 might be due to the improvement of crystallinity of a thickness of AZO layer. This figure shows a very slight change in the resistance of the OMO film; it would occur with the 
increase of the OMO thickness from $145 \mathrm{~nm}$ and beyond. However, a significant drop can be observed when the thickness value increases from 96 to $103 \mathrm{~nm}$. As the thickness of OMO layer was very thin (96 nm), the crystallinity of the AZO grew poor as shown in Fig. 4. Poor crystallinity leads to a higher sheet resistance of the AZO layer itself. It may also affect the film quality of the Mo layer, which was deposited upon the lower AZO film. As the OMO film increased to $103 \mathrm{~nm}$, the film showed an obvious $\mathrm{ZnO}$ (002) peak, which meant better film quality of the AZO film. The improvement of crystalline leads to a decrease of grain boundary scattering and an increase of carrier lifetime [16], and consequently leads to a decrease in resistivity. So, a significant drop of sheet resistance can be seen when the thickness value increases from 96 to $103 \mathrm{~nm}$. However, the sheet resistance of the OMO shows a slight change at the thickness of the OMO increase from 145 to $197 \mathrm{~nm}$. This is due to the crystallinity of the minute OMO film changes. The decrease of the resistance of the OMO film results from the thickness effect of AZO film, so the variation of the sheet resistance is little.

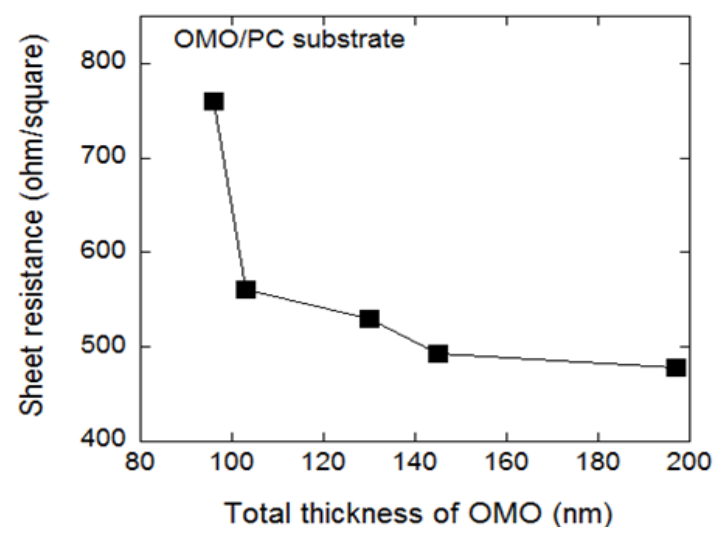

Figure 2. The sheet resistance of the OMO film with respect to different thickness of AZO.

The transmittance spectrum in the wavelength range of $300-1100 \mathrm{~nm}$ of OMO films with different thickness of AZO film is shown in Fig. 3. The red-shift effect of the increase of AZO thickness on the OMO film is found by the observation of the absorption edge and the peak value of transmittance of the spectrum. The transmittance of the OMO film increases at the range of thickness first from 96 to $130 \mathrm{~nm}$, and then decreases at the range of thickness of 148 and $197 \mathrm{~nm}$. The increase of the transmittance is due to the improvement of the AZO film quality as discussed in the XRD analysis. It is found that the crystalline of the $\mathrm{ZnO}$ (002) becomes better as the thickness of the AZO increase from 96 to $130 \mathrm{~nm}$ of the OMO film as shown in Fig. 3. In the tri-layered OMO scheme, the Mo layer acts as a reflective mirror for the incident light, and the AZO layers play the role of anti-reflective coating layer in the tri-layered films. At an appropriate thickness of AZO layers, the light beams reflected on the front and back surface of each of the AZO layers are of opposite phase and of nearly equal amplitude, which leads to the destructive interference and the reflective light is induced to diminish. Similar results have been reported by Leftheriotis et al. [17]. As the thickness of the tri-layered OMO film increases further to $197 \mathrm{~nm}$, the transmittance decreases.

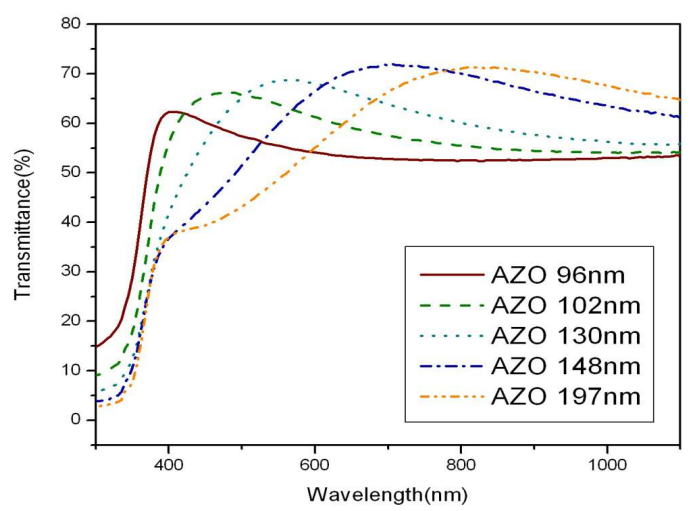

Figure 3. The transmittance of various thickness of OMO film on PC substrate

The FOM $\left(\varphi_{\mathrm{TC}}\right)$ of the AZO/Mo/AZO films as function of AZO layer thickness is calculated by using the Haacke equation [18],

$$
\phi_{T C}=\frac{T^{10}}{R_{s}}
$$

where $\mathrm{T}$ is the optical transmittance of the film at the incident wavelength of $550 \mathrm{~nm}$ and $\mathrm{R}_{\mathrm{s}}$ is the sheet resistance of the film. The FOM value of the OMO films with various AZO thicknesses is summarized at Table 2. It can be seen that a best FOM $\left(1.56 \times 10^{-5} \Omega^{-1}\right)$ is obtained at the tri-layer OMO thickness of $130 \mathrm{~nm}$. The corresponding sheet resistance and transmittance at $550 \mathrm{~nm}$ wavelength of the OMO film is $530 \Omega / \square$ and $62 \%$, respectively. At the thinner thickness of the OMO film, the high value of the FOM is due to the high value of sheet resistance of the OMO film. Increasing the AZO thickness led to a decrease in the FOM value of the OMO film due to the decreased transmission by the thicker AZO layer.

Table 2. The transmittance, FOM value of various thickness of OMO film.

\begin{tabular}{llllll}
\hline $\begin{array}{l}\text { Sputtering Time(min) } \\
\text { Avg. Transmittance }\end{array}$ & 6 & 8 & 10 & 13 & 15 \\
$(\%)$ & 55 & 60 & 62 & 61 & 54 \\
FOM $\left(\Omega^{-1}\right)$ & $3.33 \mathrm{E}-6$ & $1.08 \mathrm{E}-5$ & $1.56 \mathrm{E}-5$ & $1.45 \mathrm{E}-5$ & $4.41 \mathrm{E}-6$ \\
\hline
\end{tabular}

The crystallinity of the OMO film was observed through XRD analysis. Fig. 4 shows the XRD spectra of the OMO film with respect to various thickness of the AZO layer. The thickness of the centered Mo layer is constant and its deposition time is constant at $30 \mathrm{sec}$. The peak intensity of Mo is not detected which means the Mo layer is an amorphous structure; this may be due to the thinness of the Mo layer. The $\mathrm{ZnO}(002)$ peak at the OMO films was found as the thickness of AZO was above $102 \mathrm{~nm}$. The detecting of $\mathrm{ZnO}$ (002) phase indicates that the c-axis is 
predominantly oriented parallel to the substrate normal. Crystalline quality shows better as the thickness of AZO layer increases. Therefore, the intensity of the $\mathrm{ZnO}(002)$ peak of the tri-layers increases with the AZO layer thickness increasing. The improvement of the crystalline quality of the AZO film also decreases the resistivity of the tri-layer films as discussed in Fig. 1

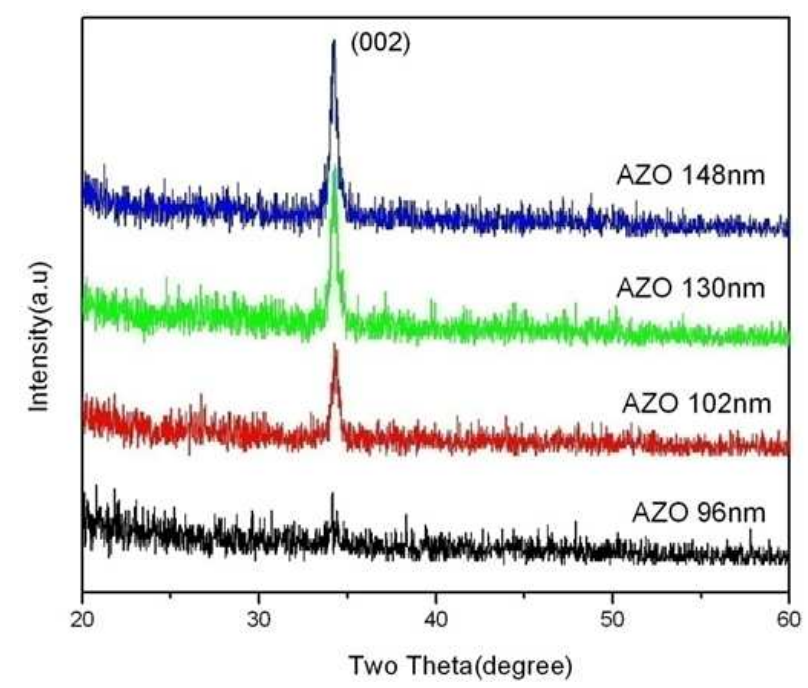

Figure 4. The XRD spectra of the OMO films.
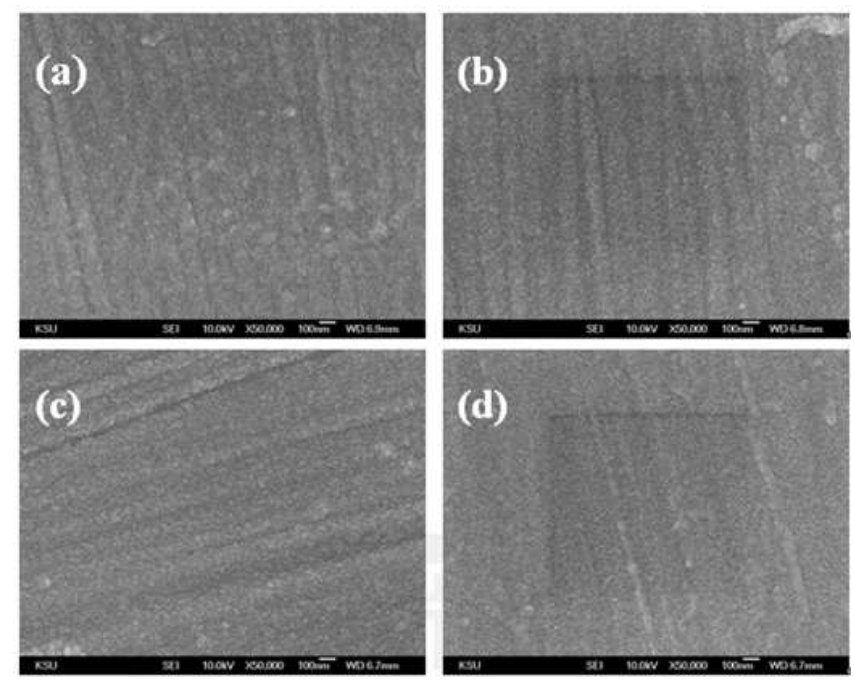

Figure 5. The SEM pictures of the OMO films on PC substrate with various thickness of OMO films (a) $96 \mathrm{~nm}$ (b) $103 \mathrm{~nm}$ (c) $130 \mathrm{~nm}$ (d) 145 $\mathrm{nm}$.

The surface morphology of the tri-layer OMO films is shown in Figs. 5(a) (d). The columnar roughness on each picture is attributed to the thermal effect on PC substrate during deposition. Surface roughness of small spherical humps is obvious at the OMO sample of $96 \mathrm{~nm}$ thickness. The roughness is due to the short deposition time of the OMO layer. It does not provide sufficient time for them to reach its suitable position. Correlating the rough surface morphology and the poor crystallinity of the sample of 96 $\mathrm{nm}$ as shown in Fig. 4, it depicts poor sheet resistance. As the thickness of the OMO film increased to $103 \mathrm{~nm}$, the spherical humps became less as shown in Fig. 5(b). This is due to the improvement of crystallinity as discussed in the $\mathrm{XRD}$ result and leading to a reduction of sheet resistance. For a further increase of the thickness of OMO film, the surface morphologies do not show obvious differences as shown in Fig. 5 (c) and (d). This is due to the growth of $\mathrm{ZnO}$ (002) which reached a saturated state as revealed in the XRD discussion.

\section{Conclusion}

The triple-layered transparent conductive oxide thin films, $\mathrm{AZO} / \mathrm{Mo} / \mathrm{AZO}$ (OMO) structure is present in the paper. The OMO film is deposited on polycarbonate (PC) substrates by a the co-sputtering process at room temperature. The thickness effect of the $\mathrm{Al}$ doped $\mathrm{ZnO}$ (AZO) to the OMO film is stressed. The dependence of the AZO thickness of the electro-optical characteristic is not obvious. A best figure of merit (FOM) of $1.56 \times 10^{-5} \Omega^{-1}$ with a sheet resistance of 530 $\Omega / \square$ and average transmittance of $62 \%$ is found at the thickness of AZO when it is equal to $130 \mathrm{~nm}$. The improvement of FOM value is a result of the reduction of sheet resistance through the addition of metal Mo layer and only gives off little transparency at this thickness of the AZO layer.

\section{Acknowledgements}

The authors would like to acknowledge the financial support of the National Science Council (NSC 101-2221-E-168-018).

\section{References}

[1] M. Purica, E. Budianu, E. Rusu, M. Danila, R. Gavrila,"Optical and structural investigation of $\mathrm{ZnO}_{-}$thin films_prepared by chemical vapor deposition (CVD)", Thin Solid Films 403/404 (2002) 485-488.

[2] G. A. Hirata, J. Mckittrik, T. Cheeks, J. M. Siqueiros, J. A. Diaz, O. Contreras, O. A. Lopez, "Synthesis and optelectronic characterization of gallium doped zinc oxide transparent electrodes", Thin Solid Films 288 (1996) 29-31.

[3] B. H. Choi, H. B. Im," Optical and electrical properties of $\mathrm{Ga}_{2} \mathrm{O}_{3}$-doped $\mathrm{ZnO}$ films prepared by r.f. sputtering", Thin Solid Films 193/194 (1990) 712-720.

[4] T. Minami, H. Sato, H. Nanto, S. Takata," High conductive and transparent silicon doped zinc oxide thin films prepared by RF magnetron sputtering”, Jpn. J. Appl. Phys. 125 (1986) L776.

[5] T. C. Lin, W. F. Huang, W. C. Huang," The effect of hydrogen annealing on the molybdenum doped $\mathrm{ZnO}$ thin film", J Mater Sci: Mater Electro, 25 (2014) 2138-2142.

[6] T. C. Lin, W.C. Huang, C. H. Liu. S. C. Chang," Structural,

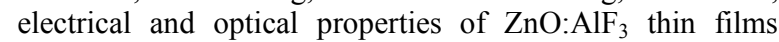
deposited by RF magnetron sputtering”, Appl. Surf. Sci., 258/7 (2012)3302-3308. 
[7] F. Ruffino, E. Carria, S. Kimiagar, I. Crupi, F. Simone, M.G. Grimaldi,"Formation and evolution of nanoscale metal structures on ITO surface by nanosecond laser irradiations of thin Au and Ag films", Sci. Adv. Mater. 4 (2012) 708-718.

[8] F. Ruffino, I. Crupi, F. Simone, M.G. Grimaldi, Formation and evolution of self-organized $\mathrm{Au}$ nanorings on indium-tin-oxide surface", Appl. Phys. Lett. 98 (2011) 023101-023103.

[9] S. Song, T. Yang, M. Lv, Y. Li, Y. Xin, L. Jiang, Z. Wu, S. Han, "Effect of $\mathrm{Cu}$ layer thickness on the structural, optical and electrical properties, of $\mathrm{AZO} / \mathrm{Cu} / \mathrm{AZO}$ tri-layer films", Vacuum 85 (2010) 39-44.

[10] D. Kim," Low temperature deposition of transparent conducting ITO/Au/ITO films by reactive magnetron sputtering”, Appl. Surf. Sci. 256 (2010) 1774-1777.

[11] J. A. Jeong, Y. S. Park, H. K. Kim, " Comparison of electrical, optical, structural, and interface properties of IZO-Ag-IZO and IZO-Au-IZO multilayer electrodes for organic photovoltaics", J. Appl. Phys. 107 (2010) 023111.

[12] Y. S. Kim, J. H. Park, D. H. Choi, H. S. Jang, J. H. Lee, H. J. Park, J. I. Choi, D. H. Ju, J. Y. Lee, D. Kim," ITO/Au/ITO multilayer thin films for transparent conducting electrode applications“"Appl. Surf. Sci. 254 (2007) 1524-1527.
[13] L. Gong, J. Lu, Z. Ye, Transparent conductive Ga-doped $\mathrm{ZnO} / \mathrm{Cu}$ multilayers prepared on polymer substrates at room temperature" Sol. Energ. Mat. Sol. C., 95 (2011)1826-1830.

[14] D. R. Sahu, J. L. Huang, "Dependence of film thickness on the electrical and optical properties of $\mathrm{ZnO}-\mathrm{Cu}-\mathrm{ZnO}$ multilayers" Appl. Surf. Sci. 253 (2006) 915-918.

[15] M. Bender, W. Seelig, C. Daube, H. Frankenberger, B. Ocker, J. Stollenwerk, "Dependence of oxygen flow on optical and electrical properties of DC-magnetron sputtered ITO films", Thin Solid Films, 326(1998)72-77.

[16] X. T. Hao, J. Ma, D. H. Zhang, T. L. Yang, H. L. Ma, Y. G. Yang, C. F. Cheng, J. Huang, Appl. Surf. Sci. 183 (2001) 137-142.

[17] G. Leftheriotis, P. Yianoulis, D. Patrikios,” Deposition and optical properties of optimised $\mathrm{ZnS} / \mathrm{Ag} / \mathrm{ZnS}$ thin films for energy saving applications", Thin Solid Films 306(1997) 92-99.

[18] G. Haacke, J. Appl. Phys. 47 (1976) 4086-4089. 